\title{
Inter-relationship among myasthenia gravis, WHO histology, and Masaoka clinical stage and effect on surgical methods in patients with thymoma: a retrospective cohort study
}

\author{
Jianfei Shen ${ }^{1 \#}$, Hongtao Tie ${ }^{2 \#}$, Anyi Xu ${ }^{3}$, Dan Chen ${ }^{2}$, Dehua Ma ${ }^{1}$, Bo Zhang ${ }^{1}$, Chengchu Zhu ${ }^{1}$, Qingchen Wu ${ }^{2}$ \\ ${ }^{1}$ Department of Thoracic Surgery, Taizhou Hospital of Zhejiang Province, Wenzhou Medical University, Linhai 317000, China; ${ }^{2}$ Department of \\ Cardiothoracic Surgery, The First Affiliated Hospital of Chongqing Medical University, Chongqing 400042, China; ${ }^{3}$ Department of Emergency \\ Medicine, Taizhou Hospital of Zhejiang Province, Wenzhou Medical University, Linhai 317000, China \\ Contributions: (I) Conception and design: C Zhu, Q Wu; (II) Administrative support: None; (III) Provision of study materials or patients: None; (IV) \\ Collection and assembly of data:H Tie, J Shen; (V) Data analysis and interpretation: D Chen, D Ma, B Zhang; (VI) Manuscript writing: All authors; \\ (VII) Final approval of manuscript: All authors. \\ "These authors contributed equally to this work. \\ Correspondence to: Chengchu Zhu. Department of Thoracic Surgery, Taizhou Hospital of Zhejiang Province, Wenzhou Medical University, Linhai \\ 317000, China. Email: zhucc@enzemed.com; Qingchen Wu. Department of Cardiothoracic Surgery, The First Affiliated Hospital of Chongqing \\ Medical University, Chongqing 400042, China. Email: qcwucq@163.com.
}

Background: The aim of study is to analyze the inter-relationship among WHO histology, myasthenia gravis (MG) and Masaoka stage and to assess the feasibility of thoracoscopic surgery in thymoma patients.

Methods: Data from 142 consecutive thymoma patients from January 2009 to March 2016 were retrospectively reviewed in our institution. Histological classification and clinical staging were assessed by WHO histology criteria and Masaoka stage. We investigated the clinical characteristics, inter-relationship among WHO histology, MG and Masaoka stage, and compared the feasibility and safety of thoracoscopic thymectomy by comparison of open thymectomy.

Results: Among 142 patients, the incidence of MG was 29.6\%. Compared with A and AB-type thymomas, a higher prevalence of advance clinical stage was in B1 to C-type thymomas (37/63 vs. 9/43, $\mathrm{P}<0.001)$, and there was an increased trend of Masaoka stage from A to $\mathrm{C}$-type thymomas $(\mathrm{P}<0.001)$. The incidence of MG was significantly higher in $\mathrm{AB}, \mathrm{B} 1$ and $\mathrm{B} 2$-type thymomas than other type thymomas (23/63 vs. $6 / 44, \mathrm{P}=0.009)$ and in early Masaoka clinical stage than advanced Masaoka clinical stage (29/80 vs. $12 / 59$, $\mathrm{P}=0.042)$. Thoracoscopic surgery could significantly decrease blood loss in patients with $(104.06 \pm 137.36$ vs. $350.91 \pm 560.79 \mathrm{~mL}, \mathrm{P}=0.001)$ or without $\mathrm{MG}(91.90 \pm 77.70$ vs. $266.32 \pm 292.60 \mathrm{~mL}, \mathrm{P}=0.02)$, with comparable complications. Additionally, thoracoscopic surgery could achieve an equal effect on the remission of MG with open surgery (7/11 vs. 10/14, $\mathrm{P}=1.000)$, and Masaoka stage was significantly associated with the remission of MG after thymectomy.

Conclusions: Our study suggests that WHO histology, MG, and Masaoka stage interrelate with one another, and Masaoka stage is an important prognostic factor in remission of MG after thymectomy in thymoma patients. Thoracoscopic thymectomy could achieve an equal efficacy to open thymectomy and should be recommended as a routine surgery for patients with early Masaoka stage.

Keywords: Thymoma; myasthenia gravis (MG); thoracoscopic thymectomy; open thymectomy

Submitted Mar 24, 2018. Accepted for publication Mar 29, 2018.

doi: $10.21037 /$ jtd.2018.05.30

View this article at: http://dx.doi.org/10.21037/jtd.2018.05.30 


\section{Introduction}

Thymoma is the most common primary neoplasm in the anterior mediastinum, which originally arises from the thymus gland. Though it accounts for $47 \%$ of tumors in the anterior mediastinum, its incidence is only about $3.2 / 100,000$ people $(1,2)$. However, it draws intensive concern for their interesting features of indolent growth pattern and characteristic of a malignant tumor of local invasion, pleural metastasis, and even systemic metastases (3). Moreover, it is associated with several autoimmune diseases, including myasthenia gravis (MG), which additionally confuses the already complicated scenario (3). However, their low incidence contributes a lack of adequate evidence to achieve a consensus regarding optimal management (4), and studies on thymoma are still needed.

Many histological classification systems of thymoma have been used owing to their widely varied histologic appearance, and the most commonly used one is the World Health Organization (WHO) histological classification $(5,6)$. Thymoma is classified into five types (types $\mathrm{A}, \mathrm{AB}$, $\mathrm{B} 1, \mathrm{~B} 2$, and B3) and thymic carcinoma according to WHO histological classification. Additionally, another widely used clinical staging system was proposed by Masaoka and colleagues (7), according to the invasion and anatomic extent of involvement via clinical and histopathologic examination. Previous studies have demonstrated that both WHO histology and Masaoka stages are significantly associated with the prognosis after thymectomy (3,8-13), and thymoma with MG or not has a controversial effect upon the prognosis $(14,15)$. However, the inter-relationship among WHO histology, MG, and Masaoka stage are rarely studied, and many efforts are necessary to explore this issue.

Surgical resection via median sternotomy remains the baseline choice for thymoma therapy. Recent years, thoracoscopy has been increasingly adopted in thoracic surgery, including mediastinal disease, and it was also introduced to thymectomy. Since the extent of resection is an important prognostic factor and completeness of resection is always being aimed. Given concern regarding completeness of resection, this minimally invasive approach has not been widely approved (16). Two meta-analyses $(17,18)$ demonstrated that thoracoscopic thymectomy could achieve an equal surgical efficacy to open thymectomy in patients with MG; however, inconsistencies still existed in some clinical outcomes. Therefore, researches are required to evaluate thoracoscopic approaches for the thymoma resection.

The present retrospective cohort study aimed to analyze the inter-relationship among WHO histology, MG, and
Masaoka stage and evaluated the feasibility and safety of thoracoscopic thymectomy by comparison of open thymectomy in patients with or without MG. Our study has two hypotheses, as follows: (I) WHO histology, MG, and Masaoka stage interrelate with one another, and both WHO histology and Masaoka stage are important prognostic factors in remission of MG; (II) thoracoscopic thymectomy could achieve an equal efficacy to open thymectomy with additional clinical advantages.

\section{Methods}

\section{Study population and data collection}

Institutional review board and informed consent were waived for the retrospective nature of the current study, and our study was reported according to the STROCSS criteria (19). This study was registered at ResearchRegistry.com as researchregistry2904. Patients with a discharge diagnosis of thymoma were retrieved in the electronic medical record system and archive documents of our institution by a professional researcher (from January 2008 to March 2016). This system includes details of patients' baseline characteristics, radiography and laboratory results, surgical procedures, and the detailed medical record from admission to discharge. Inclusion criteria: (I) patients with histological confirmation of thymoma; (II) patients undergoing surgery, including extended thymectomy, thymothymectomy, and thymoma resection, biopsy, and biopsy+ iodine 125 implant; (III) essential data could be obtained from the electronic medical record system and archive documents. Exclusion criteria: (I) patients without histopathologic record even if they diagnosed as thymoma while discharge; (II) patients with vital information unavailable. Patients' baseline characteristics, surgical records, and perioperative variables were collected by using a standard extraction form. Additionally, the prognosis of MG was obtained retrospectively by interviewing to patients or family members via telephone after the medical information collected.

\section{WHO histological classification and clinical staging}

Thymoma was diagnosed by pathologists via reviewing the Hematoxylin-eosin-stained formalin-fixed paraffin sections of thymoma specimens. Histological classification of thymoma was obtained from the records of histopathologic test, which was defined by pathologists according to WHO criteria $(5,6)$. The WHO criterion of thymoma was originally raised 
in 1999 and revised in 2004, as the following six categories: types A, AB, B1, B2, B3, and C. The revision one eliminated type $\mathrm{C}$ due to its malignant morphologic characterization and primary lesion outside the thymus. C-type thymoma, namely thymic carcinoma, was also included in our study. The WHO histological classification could not be obtained if the WHO histology not mentioned in the histopathologic report. Clinical staging of thymoma was decided on the basis of surgery record and histopathologic report according to the Masaoka system (7), which was the most important prognostic factor in thymoma and based upon the integrity of capsule, invasion into adjacent organs, and distant metastasis. Masaoka stage I-II means early clinical stages and Masaoka stage III-IV means advanced clinical stages. In our study, Masaoka stage was evaluated by two thoracic surgeons, and the inconsistency was judged by the third one. Histological types and clinical stages were regarded as categorical variables in our study.

\section{Surgical techniques and prognosis}

Surgeries for thymomas resection were all performed by associate senior doctors, and thoracoscopic thymectomy and open thymectomy were both used in our study. The surgical procedures were classified into three groups according to the completeness of resection, as follows: subtotal resection (no tumor remained macroscopically after extended thymectomy), partial resection (including tumor remained macroscopically but unresectable because of invasion to vital organ), and inoperable (including exploratory thoracotomy, simple biopsy, and iodine-125 implant surgery). The resection extent included extended thymectomy (resection of thymoma, thymus, and anterior mediastinal adipose tissue around the thymus), thymothymectomy (resection of thymoma and thymus), and thymoma resection (resection of tumor and part of thymus). In thymoma patients with MG, extended thymectomy was routinely used, while thymothymectomy or thymoma resection was used for patients without MG according to patients' conditions. Additionally, since thoracoscopic surgery was a relatively new technique and being skilled used by some of the associate senior doctors, therefore, thoracoscopic surgery or open surgery was performed depended on associate senior doctors' assessments, patients' wishes, conditions, and imaging manifestations. The evaluation of $M G$ remission after surgery was identified by criteria from Monden and colleagues (20), as follows: remission (no symptoms or minimal residual symptoms with full working life and without any medication), improved (symptoms of MG being improved controlled with same or less medication), stable (clinical characteristics unchanged), worse (more medication or worse symptoms), and death related to MG.

\section{Statistical analyses}

Data was presented as means \pm SD for continuous variables and frequencies for categorical variables. Comparisons of different groups were performed by using Pearson's $\chi^{2}$-test for categorical variables, unpaired $t$-test (two groups) and ANOVA (more than two groups) for continuous variables. All the statistical analysis was based on the patients with available information, and patients with missing data were not included individual analysis. All statistical analyses were performed using the software SPSS Statistics 21.0. A P value of less than 0.05 was considered statistical significant.

\section{Results}

\section{Baseline characteristics}

The flow chart of patients selection process and the total numbers of patients with WHO histology, MG, and Masaoka stage are presented in Figure 1. Table 1 illustrates the basic characteristics of the 142 included patients, including 76 men and 66 women with an average age of $51.35 \pm 14.30$ years, and MG was presented in 42 patients (29.6\%). Masaoka stage could be obtained from 139 patients by reviewing the surgery records and examination results, and WHO histological classification could be adopted in 107 patients. Among the 142 patients, 32 (22.5\%) presented the symptoms of chest tightness or chest pain, $30(21.1 \%)$ presented cough, $3(2.1 \%)$ presented with fever, and 4 (2.8\%) presented edema. Grouping patients according to Masaoka stage and MG, the symptoms of chest pain/chest tight and cough were significantly higher in patients with advanced clinical stage and no MG. Additionally, incidence of chest pain/chest tight and cough was significantly different among the six WHO histology.

\section{Inter-relationship between WHO bistology, Masaoka stage and $M G$}

Table 2 describes the distribution of WHO histology and Masaoka stage in 106 patients. In A and $\mathrm{AB}$ type thymomas, the prevalence of early clinical stages was significantly higher than B1 through C type thymomas (34/43 vs. 26/63; 


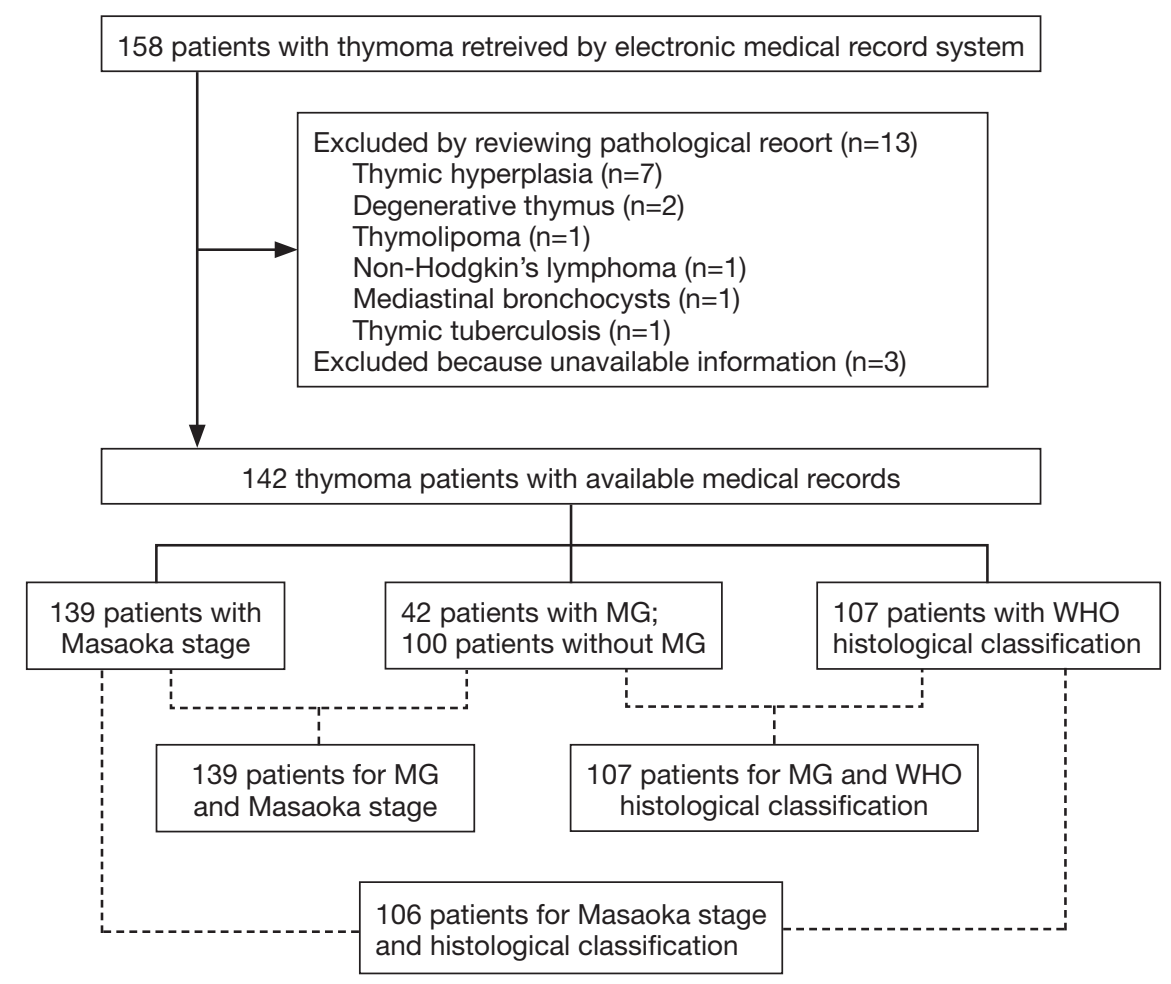

Figure 1 The flow chart of patients selection process.

$\mathrm{P}<0.001)$. While the prevalence of advanced clinical stage was observed higher in $\mathrm{B} 1$ through $\mathrm{C}$ type thymomas, compared with $\mathrm{A}$ and $\mathrm{AB}$ type thymomas (37/63 vs. 9/43; $\mathrm{P}<0.001)$. The overall results show that there is an increased trend of Masaoka stage from A to $C$ type thymomas $(\mathrm{P}<0.001)$. The distribution between MG and Masaoka stage in 139 patients is also presented in Table 2, and no association was observed among them $(\mathrm{P}=0.120)$. However, early clinical stage was associated with a significant increased incidence of MG (29/80 vs. $12 / 59, \mathrm{P}=0.042)$. Table 2 shows the correlation between MG and WHO histology in 107 participants. Overall, a significant association was observed $(\mathrm{P}=0.034)$. In detail, the incidence of $\mathrm{MG}$ in $\mathrm{AB}$, $\mathrm{B} 1$ and $\mathrm{B} 2$-type thymomas is significantly higher than other three types $(23 / 63$ vs. $6 / 44, \mathrm{P}=0.009)$.

\section{Association between surgical methods, completeness of resection and Masaoka stage}

Table $S 1$ outlines the basic characteristics of the 136 patients undergoing surgery according to surgical methods. Table 3 shows the distribution of surgical methods, completeness of resection and Masaoka stage in 136 thymoma patients undergoing surgery, with 66 by open surgery and 70 by thoracoscopic surgery. No difference in completeness of resection was observed between thoracoscopic surgery and open surgery $(\mathrm{P}=0.396)$. As for the correlation between surgical methods and Masaoka stage, a significant association was observed $(\mathrm{P}=0.004)$. Specifically, a significantly increased prevalence of early clinical stages was found in the thoracoscopic surgery group (51/70 vs. $29 / 66, \mathrm{P}=0.001$ ), while a significantly increased prevalence of advance clinical stages in the open surgery group (19/70 vs. 37/66; $\mathrm{P}=0.001$ ). Moreover, six IV stage patients underwent thoracoscopic surgery only for biopsy but not resection. As for the correlation between Masaoka stage and completeness of resection, a significant inter-relationship between clinical stage and rate of resection incompleteness existed $(\mathrm{P}<0.001)$, and the incidence of unresectable cases was significantly higher in advanced clinical stage (22/56 vs. 0/80; $\mathrm{P}<0.001)$.

\section{Clinical outcomes between open and thoracoscopic surgery}

Table 4 presents the clinical outcomes between open surgery and thoracoscopic surgery. Compared with open surgery, 


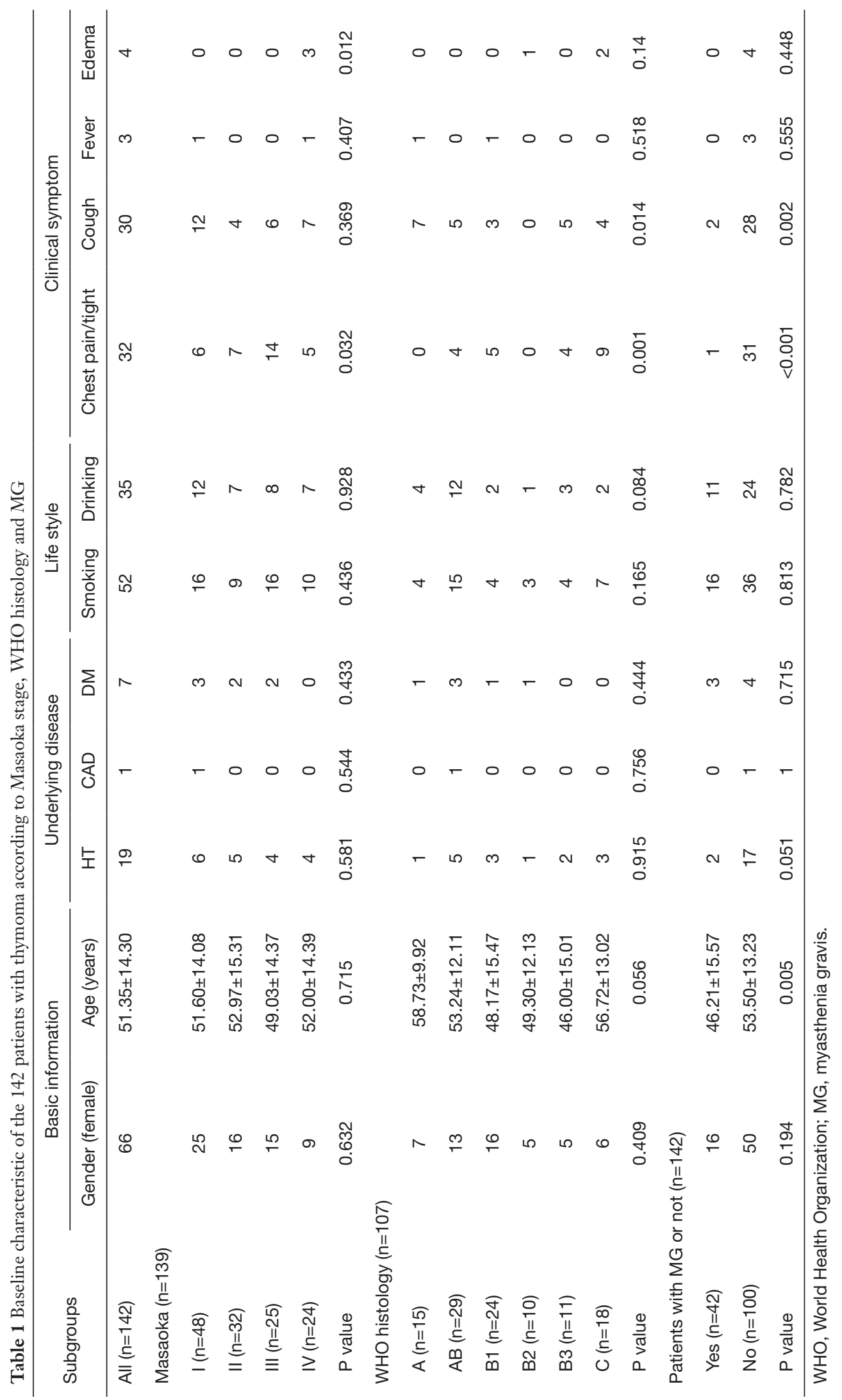


Table 2 Inter-relationship among Masaoka stage, WHO histology and MG

\begin{tabular}{|c|c|c|c|c|c|c|c|c|}
\hline Subgroups & \multicolumn{4}{|c|}{ Masaoka stage } & $P$ value & \multicolumn{2}{|c|}{ MG } & $P$ value \\
\hline WHO histology & & & & & $<0.001$ & & & 0.034 \\
\hline$A$ & 7 & 4 & 3 & 0 & & 3 & 12 & \\
\hline$A B$ & 16 & 7 & 4 & 2 & & 10 & 19 & \\
\hline B2 & 1 & 3 & 4 & 2 & & 6 & 4 & \\
\hline B3 & 2 & 1 & 3 & 5 & & 2 & 9 & \\
\hline C & 0 & 1 & 8 & 9 & & 1 & 17 & \\
\hline MG & & & & & 0.12 & & & - \\
\hline
\end{tabular}

WHO, World Health Organization; MG, myasthenia gravis.

Table 3 Association among Masaoka stage, surgical methods and completeness of resection

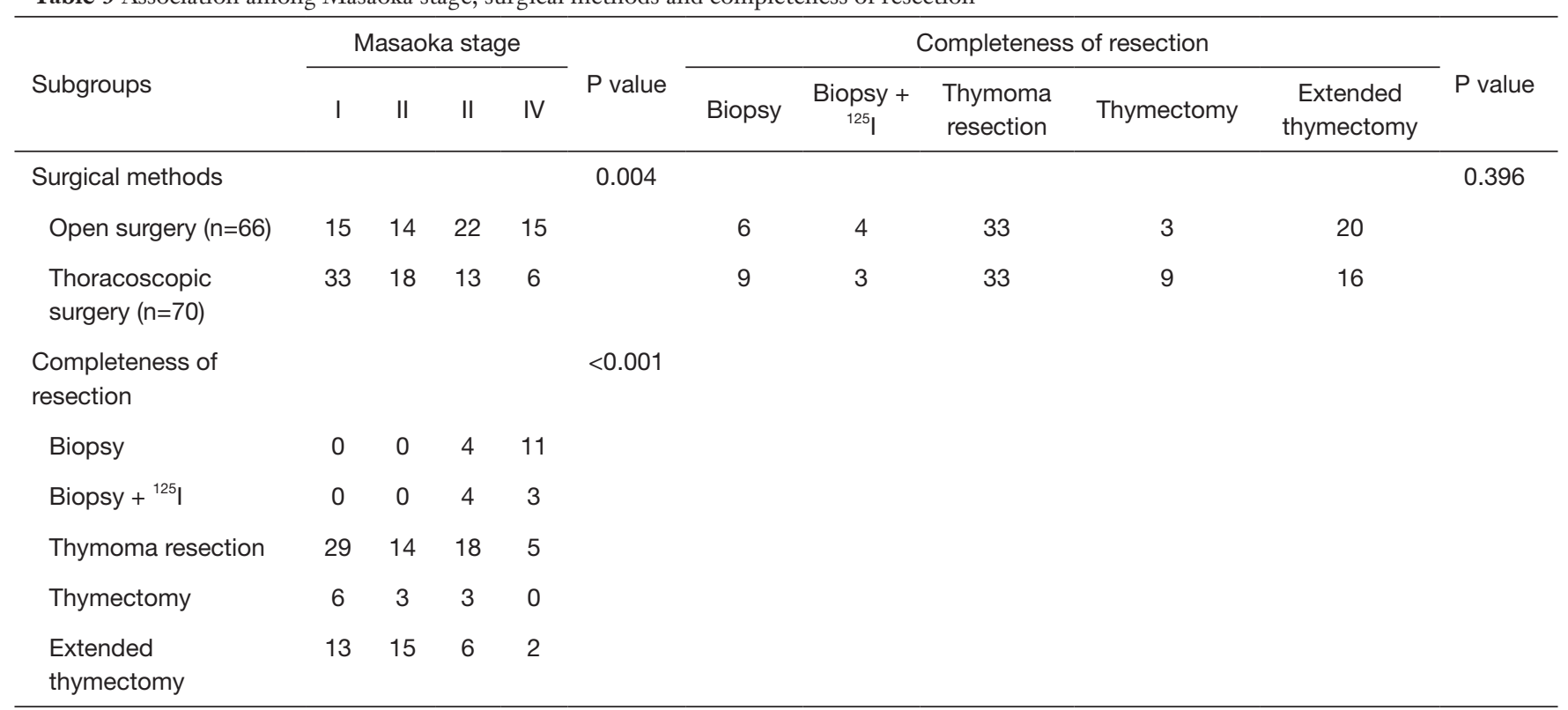

thoracoscopic surgery could significantly decrease the blood loss $(\mathrm{P}<0.001)$, without differences in operation time, mechanical ventilation, myasthenia crisis, tracheotomy and perioperative mortality by comparison with open surgery (all $\mathrm{P}>0.05$ ). Additionally, the results remained similar while the only MG patients were involved.

\section{Prognosis of the patients with MG after surgery}

Table 5 shows the prognosis of patients with MG. Twentyfive of the $40 \mathrm{MG}$ patients could be retrospectively studied. The symptoms of MG disappeared in ten patients, and the time to recovery ranged from 1 to 24 months after discharge, with a median of 15.7 months. In the remaining 
Table 4 Perioperative outcomes of the patients undergoing surgery

\begin{tabular}{|c|c|c|c|c|c|c|}
\hline Outcomes & \multicolumn{3}{|c|}{ Total patients undergoing thymectomy $(n=136)$} & \multicolumn{3}{|c|}{ MG Patients undergoing surgery $(n=40)$} \\
\hline Operation time (min) & $143.24 \pm 64.17$ & $131.11 \pm 62.48$ & 0.272 & $144.37 \pm 40.57$ & $149.38 \pm 69.93$ & 0.781 \\
\hline MV duration (min) & $356.00 \pm 477.93$ & $344.70 \pm 576.29$ & 0.909 & $697.35 \pm 704.47$ & $862.31 \pm 962.24$ & 0.577 \\
\hline Tracheotomy & 2 & 3 & 1 & 2 & 3 & 1 \\
\hline Mortality & 0 & 0 & - & 0 & 0 & - \\
\hline
\end{tabular}

MG, myasthenia gravis; MV, Mechanical ventilation.

Table 5 Prognosis of 25 patients with MG according to WHO histology, Masaoka stage and surgery methods

\begin{tabular}{|c|c|c|c|c|}
\hline Subgroups & $\mathrm{n}$ & Improved & Stable & $P$ value \\
\hline Masaoka stage & 25 & & & 0.001 \\
\hline I & 11 & 9 & 2 & \\
\hline II & 7 & 7 & 0 & \\
\hline III & 4 & 0 & 4 & \\
\hline IV & 2 & 1 & 2 & \\
\hline $\begin{array}{l}\text { WHO histologic } \\
\text { type }\end{array}$ & 21 & & & 0.709 \\
\hline A & 2 & 2 & 0 & \\
\hline$A B$ & 8 & 5 & 3 & \\
\hline B1 & 4 & 3 & 1 & \\
\hline B2 & 5 & 3 & 2 & \\
\hline B3 & 2 & 1 & 1 & \\
\hline Surgical methods & 25 & & & 1.000 \\
\hline Open surgery & 11 & 7 & 4 & \\
\hline $\begin{array}{l}\text { Thoracoscopic } \\
\text { surgery }\end{array}$ & 14 & 10 & 4 & \\
\hline
\end{tabular}

WHO, World Health Organization; MG, myasthenia gravis.

15 patients, the symptoms of MG ameliorated in seven patients and remained stable in eight patients, and deteriorated in none of them, with a total effective rate of $68 \%$. The improved rate of MG symptom, including MG symptom disappearance and amelioration, was significantly associated with Masaoka stage $(\mathrm{P}=0.001)$, but not WHO histology $(\mathrm{P}=0.709)$ or surgical methods $(\mathrm{P}=1.000)$.
Moreover, early clinical stage was associated with higher incidence of improved MG symptom than advanced clinical stage $(16 / 18$ vs. $1 / 7 ; \mathrm{P}=0.002)$.

\section{Discussion}

In the current study, we retrospectively investigated the clinical characteristics, inter-relationship among WHO histology, MG and Masaoka stage, and comparison of surgical methods in a series of patients with thymoma. In our study, the incidence of MG was $29.6 \%$, which was in accordance with the previous study (14), and symptoms of chest pain/chest tight and cough were more common in patients with advanced clinical stage and no MG. Additionally, WHO histology, MG and Masaoka stage interrelated with one another. In detailed, there was an increased trend of Masaoka stage with thymomas type from A to $C$, and the incidence of $M G$ was significantly higher in $\mathrm{AB}, \mathrm{B} 1$ and $\mathrm{B} 2$-type thymomas than other types and in early Masaoka stage than advanced Masaoka stage. Thoracoscopic surgery could significantly decrease blood loss in thymoma patients with or without MG, with comparable complications and clinical outcomes. For the prognosis of MG, Masaoka stage, but not surgical methods and WHO histology was the important prognostic factor.

The association between epidemiologic features among patients with or without MG is similar to the results from previous studies $(21,22)$ that no association exists between MG and demographic characteristics in thymoma patients, and this null association sustains when grouping by WHO histology and Masaoka stage. Additionally, living habits are also similar in patients with or without MG, of different WHO histology and different Masaoka stage. Common 
symptoms of thymoma include chest pain, chest tight, cough, and swallowing difficulty due to compression or invasion of the tumor. In our study, the most common symptoms were chest pain, chest tight and cough, and all of them were found to be more common in patients without MG and in advanced clinical stage. Therefore, clinical symptoms of chest pain/chest tight and cough, rather than demographic characteristics or living habit, might give a good indication of the patients characteristic of MG and Masaoka stage.

The inter-relationship drawn from our study is that $\mathrm{MG}$ is associated with early clinical stage and WHO histology of $\mathrm{AB}, \mathrm{B} 1$ and $\mathrm{B} 2$-type thymomas. The results are consistent with the previous study $(14,23)$. High prevalence of $M G$ in early clinical stages might be attributed to the early discovery thymoma because of the MG symptom, and the favorable prognosis of thymoma patients with $M G$ also supports the current finding $(14,24)$. However, some documents argued that MG was correlated with only B1 and $\mathrm{B} 2$ rather than B3 type thymomas $(25,26)$. Consistently, the incidence of MG in B1 and B2 type thymomas is much higher than B3, and this finding was similar to the study by Okumura (23). It is speculated that T-lymphocytes development is involved in the pathogenesis of MG (3). Therefore, the different proportion of T-lymphocytes contributes the different prevalence of MG in patients with different WHO histology. Additionally, an increased trend of Masaoka stage exists when WHO histology moving from A to $\mathrm{C}$ type thymomas, owing to the increasing malignancy of thymoma from A to C type.

With the advent and advancement of video-assisted thoracoscopic surgery in thoracic surgery, some previous studies $(17,18,27-29)$ have been performed to compare the safety and efficacy of thoracoscopic surgery with open surgery for thymectomy. Consistent with our findings, Odaka and colleagues (29) demonstrated that Masaoka stage played a decisive role in surgical methods selection. In detail, patients with advanced clinical stage are preferred to receive open thymectomy, and the completeness of thymoma resection is similar in both surgical groups. Thoracoscopic thymectomy has an equal effect on longterm oncologic outcomes with open thymectomy, with similar 5-year overall survival rate, 5-year disease-free survival rate and 5-year recurrence-free survival rate between the two groups $(28,29)$. Moreover, thoracoscopic thymectomy was associated with less blood loss, lower blood transfusion rate, decreased inflammatory cytokine response, shorter hospital length of stay, earlier removal of chest drains, and superior cosmesis $(17,18,28,30,31)$. Additionally, the efficacy and advantages of thoracoscopic thymectomy sustained in patients with MG, as also confirmed by previous studies (32). Theoretically, thoracoscopic thymectomy could remove thymic tissue more completely under the advantages of better exposure, superior illumination and magnification (30). Therefore, thoracoscopic thymectomy could be an alternative method for thymoma patients with early Masaoka stage despite the presence or absence of MG and WHO histology. However, though a very rare incidence, some catastrophic complications such as major vascular injuries should be concerned in thoracoscopic thymectomy (33).

For thymoma patients with MG, the completeness of thymic tissue resection and clearance significantly affects the MG remission (34). In our study, the incidence of improved disease of $M G$ was $68 \%$ in all patients and $71.4 \%$ in the thoracoscopic group, which was comparable with the previous report (35), and thoracoscopic surgery has a slightly superior efficacy on MG remission. Based on current evidence and our results, we could conclude that thoracoscopic thymectomy should be recommended as a routine surgery for thymoma patients with early Masaoka stage. Though WHO histology and clinical stage are associated with the incidence of MG, Masaoka stage, but not WHO histology, had an evident effect on the remission of the MG symptom. Thus, Masaoka stage is more serviceable than WHO histology to predict the prognosis of MG after thymectomy.

Some advantages exist in our study. Firstly, all research data except the remission of MG were based on medical records, which were relatively reliable and could substantially eliminate the interview bias. Secondly, we not only compared thoracoscopic thymectomy and open thymectomy, but also the potential influence factor of WHO histology, Masaoka stage and MG on surgical methods selection and prognosis of MG were also assessed. Thirdly, we compared the two surgical methods in both patients with $M G$ and without MG, and the comparison in patients with $M G$ was a matter of especial interest because of the conventional view that poor prognosis of MG might be induced by thoracoscopic thymectomy for the incompleteness of thymoma tissue. Meanwhile, limitations should also be considered. Because of the essence of a retrospective study, the interview bias about MG remission might exist, and some research data was unavailable. However, only few of our research data and patients were missed, and the results from our study share 
the same idea with the previous evidence. Additionally, surgeons' subjective factors were involved in the surgery method selection, which might cause the baseline incomparability between thoracoscopic surgery and open surgery groups. Though no differences among the baseline characteristics were observed in the two groups (Table S1), other clinical factors, except the analyzed Masaoka stage and completeness of resection, should also be concerned. Moreover, oncological outcomes were not analyzed in our study. As vital clinical interest in thymoma patients, oncological outcomes should be concerned about in further researches.

In conclusion, our study suggests that WHO histology, MG and Masaoka stage interrelate with one another, and Masaoka stage is associated with the prognosis of MG symptom. Thoracoscopic thymectomy could achieve an equal efficacy in patients with or without MG, and it should be recommended as a routine surgery for patients with early Masaoka stage. Oncological outcomes should be focused in future researches to further evaluate the feasibility of thoracoscopic surgery for thymoma patients.

\section{Acknowledgements}

We thank Feng He, Xiao-Long Chen and Jian-Qiong Xiong for their help to search archive document and collect the data. Funding: This project was supported by the Zhejiang Provincial Natural Science Foundation of China (No. LQ18H160029) and Taizhou Postdoctoral Science Foundation (No. 201702).

\section{Footnote}

Conflicts of Interest: The authors have no conflicts of interest to declare.

Ethical Statement: Institutional review board and informed consent were waived for the retrospective nature of the current study.

\section{References}

1. de Jong WK, Blaauwgeers JL, Schaapveld M, et al. Thymic epithelial tumours: a population-based study of the incidence, diagnostic procedures and therapy. Eur J Cancer 2008;44:123-30.

2. Engels EA, Pfeiffer RM. Malignant thymoma in the United States: demographic patterns in incidence and associations with subsequent malignancies. Int $\mathrm{J}$ Cancer
2003;105:546-51.

3. Ruffini E, Filosso PL, Mossetti C, et al. Thymoma: inter-relationships among World Health Organization histology, Masaoka staging and myasthenia gravis and their independent prognostic significance: a single-centre experience. Eur J Cardiothorac Surg 2011;40:146-53.

4. Liu SV, Subramaniam D, Giaccone G. Thymomas: the need for prospective studies. J Thorac Oncol 2013;8:1230-31.

5. Rosai J, Sobin LH. World Health Organization, International Histological Classification of Tumours: Histological Typing of Tumours of the Thymus, 2nd ed. Berlin: Springer, 1999.

6. Marx A, Ströbel P, Zettl A, et al. Thymomas. In: Travis WD, Brambilla E, Muller-Hermelink HK, et al. editors. World Health Organization Classification of Tumours: Pathology and Genetics of the Lung, Pleura, Thymus and Heart. Lyon, France: IARC Press, 2004.

7. Masaoka A, Monden Y, Nakahara K, et al. Follow-up study of thymomas with special reference to their clinical stages. Cancer 1981;48:2485-92.

8. Detterbeck FC. Clinical value of the WHO classification system of thymoma. Ann Thorac Surg 2006;81:2328-34.

9. Kondo K, Yoshizawa K, Tsuyuguchi M, et al. WHO histologic classification is a prognostic indicator in thymoma. Ann Thorac Surg 2004;77:1183-88.

10. Marchevsky AM, Gupta R, Casadio C, et al. World Health Organization classification of thymomas provides significant prognostic information for selected stage III patients: evidence from an international thymoma study group. Hum Pathol 2010;41:1413-21.

11. Nakagawa K, Asamura H, Matsuno Y, et al. Thymoma: a clinicopathologic study based on the new World Health Organization classification. J Thorac Cardiovasc Surg 2003;126:1134-40.

12. Okumura M, Shiono H, Minami M, et al. Clinical and pathological aspects of thymic epithelial tumors. Gen Thorac Cardiovasc Surg 2008;56:10-6.

13. Rena O, Papalia E, Maggi G, et al. World Health Organization histologic classification: an independent prognostic factor in resected thymomas. Lung Cancer 2005;50:59-66.

14. Kondo K, Monden Y. Thymoma and myasthenia gravis: a clinical study of 1,089 patients from Japan. Ann Thorac Surg 2005;79:219-24.

15. Yu L, Zhang XJ, Ma S, et al. Different characteristics of thymomas with and without myasthenia gravis. Ann Surg Oncol 2012;19:94-8. 
16. Tomaszek S, Wigle DA, Keshavjee S, et al. Thymomas: review of current clinical practice. Ann Thorac Surg 2009;87:1973-80.

17. Gung Y, Zhang H, Li S, et al. Sternotomy versus videoassisted thoracoscopic surgery for thymectomy of myasthenia gravis patients: A meta-analysis. Asian J Endosc Surg 2016;9:285-94.

18. Qi K, Wang B, Wang B, et al. Video-assisted thoracoscopic surgery thymectomy versus open thymectomy in patients with myasthenia gravis: a meta-analysis. Acta Chir Belg 2016;116:282-8.

19. Agha RA, Borrelli MR, Vella-Baldacchino M, et al. The STROCSS statement: Strengthening the Reporting of Cohort Studies in Surgery. Int J Surg 2017;46:198-202.

20. Monden Y, Nakahara K, Fujii Y, et al. Myasthenia gravis in elderly patients. Ann Thorac Surg 1985;39:433-6.

21. López-Cano M, Ponseti-Bosch JM, Espin-Basany E, et al. Clinical and pathologic predictors of outcome in thymoma-associated myasthenia gravis. Ann Thorac Surg 2003;76:1643-9.

22. Monden Y, Uyama T, Nakahara K, et al. Clinical characteristics and prognosis of myasthenia gravis with other autoimmune diseases. Ann Thorac Surg 1986;41:189-92.

23. Okumura M, Miyoshi S, Fujii Y, et al. Clinical and functional significance of WHO classification on human thymic epithelial neoplasms: a study of 146 consecutive tumors. Am J Surg Pathol 2001;25:103-10.

24. Okumura M, Miyoshi S, Takeuchi Y, et al. Results of surgical treatment of thymomas with special reference to the involved organs. J Thorac Cardiovasc Surg 1999; 117:605-13.

25. Evoli A, Minisci C, Di Schino C, et al. Thymoma in patients with MG: characteristics and long-term outcome.
Neurology 2002;59:1844-50.

26. Margaritora S, Cesario A, Cusumano G, et al. Thirty-fiveyear follow-up analysis of clinical and pathologic outcomes of thymoma surgery. Ann Thorac Surg 2010;89:245-52.

27. Fiorelli A, Mazzella A, Cascone R, et al. Bilateral thoracoscopic extended thymectomy versus sternotomy. Asian Cardiovasc Thorac Ann 2016;24:555-61.

28. Yang Y, Dong J, Huang Y. Thoracoscopic thymectomy versus open thymectomy for the treatment of thymoma: A meta-analysis. Eur J Surg Oncol 2016;42:1720-8.

29. Odaka M, Shibasaki T, Kato D, et al. Comparison of oncological results for early- and advanced-stage thymomas: thoracoscopic thymectomy versus open thymectomy. Surg Endosc 2017;31:734-42.

30. Raza A, Woo E. Video-assisted thoracoscopic surgery versus sternotomy in thymectomy for thymoma and myasthenia gravis. Ann Cardiothorac Surg 2016;5:33-7.

31. Xie A, Tjahjono R, Phan K, et al. Video-assisted thoracoscopic surgery versus open thymectomy for thymoma: a systematic review. Ann Cardiothorac Surg 2015;4:495-508.

32. Raza A, Woo E. Video-assisted thoracoscopic surgery versus sternotomy in thymectomy for thymoma and myasthenia gravis. Ann Cardiothorac Surg 2016;5:33-7.

33. Özkan B, Toker A. Catastrophes during video-assisted thoracoscopic thymus surgery for myasthenia gravis. Interact Cardiovasc Thorac Surg 2016;23:450-3.

34. Port JL, Ginsberg RJ. Surgery for thymoma. Chest Surg Clin N Am 2001;11:421-37.

35. Savcenko M, Wendt GK, Prince SL, et al. Videoassisted thymectomy for myasthenia gravis: an update of a single institution experience. Eur J Cardiothorac Surg 2002;22:978-83.
Cite this article as: Shen $\mathrm{J}$, Tie $\mathrm{H}, \mathrm{Xu} A$, Chen D, Ma D, Zhang B, Zhu C, Wu Q. Inter-relationship among myasthenia gravis, WHO histology, and Masaoka clinical stage and effect on surgical methods in patients with thymoma: a retrospective cohort study. J Thorac Dis 2018;10(5):2981-2990. doi: 10.21037/ jtd.2018.05.30 
Supplementary

Table S1 Baseline characteristic of the 136 patients undergoing surgery

\begin{tabular}{lccc}
\hline Variables & Open surgery & Thoracoscopic surgery & P value \\
\hline Gender (female/all) & $27 / 66$ & $37 / 70$ & 0.163 \\
Age (years) & $53.2 \pm 13.96$ & $49.89 \pm 14.69$ & 0.181 \\
HT & $10 / 66$ & $8 / 70$ & 0.522 \\
CAD & $0 / 66$ & $1 / 70$ & 1 \\
DM & $5 / 66$ & $2 / 70$ & 0.392 \\
Smoking & $27 / 66$ & $23 / 70$ & 0.33 \\
Drinking & $17 / 66$ & $16 / 70$ & 0.693 \\
\hline
\end{tabular}

$\mathrm{HT}$, hypertension; CAD, coronary artery disease; DM, diabetes mellitus. 